\title{
Photonics-Based Dual-Band Radar for Landslides Monitoring in Presence of Multiple Scatterers
}

\author{
Suzanne Melo , Salvatore Maresca, Sergio Pinna , Filippo Scotti , Milad Khosravanian, \\ Arismar Cerqueira S. Jr. , Filippo Giannetti , Abhirup Das Barman, and Antonella Bogoni
}

\begin{abstract}
In this paper, a dual-band photonics-based radar system used for precise displacement measures in a multitarget scenario is described. The radar was designed for monitoring applications to prevent both structural failures of buildings and landslides. The radar system exploits the technique of stepped frequency continuous wave signal modulation and the displacement of the targets is evaluated through differential phase measurements. In this work, encouraged by the results already achieved in the single-target scenario, we present an investigation extended to the case of multiple targets. We aim to evaluate the accuracy of the displacement estimation both from a simulated and experimental point of view, and to understand how multiple targets impact on the final estimate of displacements. Simulation results demonstrate that it is possible to achieve a typical accuracy of less than $0.2 \mathrm{~mm}$ for distances up to $400 \mathrm{~m}$. These results are confirmed by preliminary experimental outcomes, which take into account different operative conditions with multiple targets. Finally, concluding remarks and perspectives draw the agenda for our future investigations.
\end{abstract}

Index Terms-Interferometry, mode-locked lasers, radio frequency photonics, radar, remote sensing and sensors.

\section{INTRODUCTION}

A LARGE branch of civil engineering is interested in building integrity analysis and slope stability monitoring applications. For these, it is of crucial importance to be able to detect tiny target displacements which could be precious indications of forthcoming structural failures or landslides [1].

This task has been accomplished in many different ways over the years. For example, using extensometers, inclinometers,

This work was supported in part by the National Project PREVENTION (with the contribution of Ministry of Foreign Affairs, Directorate General for the Country Promotion) and in part by the EU projects ROBORDER \#740593. (Corresponding author: Suzanne Melo.)

S. Melo, S. Maresca, and A. Bogoni are with the Scuola Superiore Sant'Anna, 56124 Pisa, Italy (e-mail: s.assisdesouzamelo@ sssup.it; salvatore. maresca@cnit.it; antonella.bogoni@sssup.it).

S. Pinna is with the Photonics Laboratory, University of California, Santa Barbara, CA 93106 USA (e-mail: pinna@ece.ucsb.edu).

F. Scotti is with the CNIT (Consorzio Nazionale Interuniversitario per le Telecomunicazioni), 56124 Pisa, Italy (e-mail: filippo.scotti@cnit.it).

M. Khosravanian and F. Giannetti are with the Department of Information Engineering, University of Pisa, 56122 Pisa, Italy (e-mail: soroor 2069@yahoo.com; filippo.giannetti@iet.unipi.it).

A. Cerqueira S. Jr. is with the National Institute of Telecommunications (Inatel), Santa Rita do Sapucai 37540-000, Brazil (e-mail: arismar@inatel.br).

A. Das Barman is with the Institute of Radio Physics and Electronics, University of Calcutta, Kolkata 700 009, India (e-mail: abhirup1.rpe@ gmail.com). crack meters, Global Positioning System (GPS) receivers, etc. However, their ability to provide a deformation map over large areas is restricted, due to their localized operation. In this context, monitoring based on radars can be quite advantageous. Radars can largely overcome previous traditional methods in terms of remotely operation, in case of difficult and inaccessible areas, and the ability to offer deformation maps of large areas. In this context, radar differential interferometry is one of the most effective ways for measuring such structural movements, providing displacement maps with very high spatial resolution, especially for disaster prevention and risk mitigation [1], [2].

The majority of radar systems deployed for such applications are made up by airborne or spaceborne synthetic aperture radar (SAR) and by ground-based systems. SAR systems exploit the motion of the radar antenna to provide finer spatial resolution w.r.t. conventional beam-scanning radars. Typically, they are mounted on moving platforms (e.g., aircraft or satellite). Thus, the high spatial resolution obtained through SAR processing and the wide coverage area offered by the satellite platform allow to obtain high resolution displacement maps of wide land areas [3]. However, these features come at the cost of long revisiting time (no real-time monitoring on a continuous basis), unwrapping problems (i.e., false target problems), and signal phase distortions caused by the propagation in the atmosphere [4]. Conversely, ground-based systems are precious tools for monitoring smaller areas without interruption and can provide precise displacement estimates down to sub-millimeter scale at hundreds of meters up to few kilometers distance [5].

A common approach for estimating target displacements is to use differential interferometry, exploiting the SteppedFrequency Continuous Wave (SFCW) signal modulation [5]. Differently from the pulsed radar where a pulse is transmitted and time-of-flight measurements are used to determine the distance from objects, CW radars continuously broadcast radar waveforms, which may be considered to be pure sine waves, and the phase of the returning echo is used to determine range. In SFCW radars a sequence of frequency steps may be employed to extend the range. Displacement evaluation are obtained by interferometric technique, i.e., comparing the phase information of the back scattered signal collected at different times.

Initially developed to detect buried objects, SFCW radar sensors are, nowadays, widely used in a number of both civilian and military applications. In particular, detection and identification of dangerous conditions of civil strictures (e.g., bridges, buildings, buried pipes or archeological artifacts). 
In SFCW radars, a sequence in time domain of sinusoidal signals with slightly different frequencies is generated and transmitted for a fixed time, named step interval generating pulsed trains. These sinusoidal signals are coherently generated with a constant frequency separation, namely the frequency step, in a defined frequency range [6]. The total used band is given by the difference between the the minimum and maximum frequencies of sinusoids and the frequency step width are jointly chosen to satisfy a given operative unambiguous range and range resolution, as better described in Section III. Several cycles of step frequency modulation are used for each acquisition. Each sinusoid composing the SFCW signal is characterized by a very narrow instantaneous bandwidth. For this reason, this solution results in a valuable improvement of the noise figure at the receiver, thus leading to an improvement of both sensitivity and dynamic range, whilst maintaining a good average power. Moreover, by transmitting only one frequency at a time, it could be possible to properly compensate, through ad-hoc signal processing techniques, the received signals, in the case they propagate through lossy media with known characteristics, and for any non-linear effect caused by the transmitter or the receiver. Furthermore, one of the main advantages of SFCW modulation is that the signal energy is distributed in time. This feature allows to reduce the system power requirement, and it is ideal to avoid non-linear effects of the electronic components due to high power transmission [7].

Once the backscattered signal is acquired by the receiver, it is mixed with the reference signal (i.e., the transmitted signal), in order to obtain the phase information. Then, differential interferometry is used to evaluate the phase variations between two consecutive target observations. Phase information is then converted into a precise displacement measure, as will be better explained in Section III. It is straightforward that the system phase stability and the coherence among the frequency tones play a decisive role in the final accuracy of phase measurements. Therefore, special attention should be aimed to limit the phase noise in the signal generation stage, thus guaranteeing a high degree of signal coherence.

An excellent solution is based on the use of photonics. In fact, recently photonics for microwave systems have been demonstrated to guarantee a higher stability than electronic technology. In fact, photonics enable the generation and reception of Radio Frequency (RF) signals, without the need of noisy mixers and RF oscillators. Mixers are replaced by low-noise electro-optical and opto-electronic conversion blocks, while RF oscillators are substituted by highly stable optical oscillators (i.e., lasers), accordingly improving the RF signal coherence [8]. In addition, photonics help in improving the frequency flexibility of the system. In fact, with a single photonics-based transceiver (i.e., a single optical oscillator) it is possible to simultaneously generate multiple signals in different RF bands, as demonstrated in [9]. This is a very desirable feature, because it guarantees an intrinsic phase coherence among the generated multiband signals.

In this framework, our group proposed for the first time a photonics-based radar system able to exploit the combination of SFCW modulation and differential interferometry to perform high precision displacement measurements [10]. At its actual stage of development, the prototype is a dual-band radar, meaning that it can contemporaneously generate and transmit two signals, coherent with each other, and locked on two different frequency bands (S- and X-bands).

Coherent multi-band radars introduce important innovative and advantageous features. In fact, the possibility to choose different carrier frequencies makes the system tunable, and, thus, more sensitive to the different scattering properties of a given target. Moreover, the choice of multiple frequencies makes the system robust against unwanted returns from the environment. These returns, called clutter, can respond in different ways according to several factors (e.g., the size of the scatterer w.r.t. the wavelength, the geometry, etc.), generating false alarms (i.e., false targets). Weather conditions can have an impact on the received signal as well, in terms of echo attenuation or generation of false targets. At last, as known from the radar range equation, the carrier frequency has an effect on the the final reachable range of interest, due to the different absorptions and distortions experienced by the RF signal as a function of its carrier frequency [11].

Another innovative and useful feature of the photonics-based solution is the strong coherence among the generated sinusoidal tones of the SFCW signal, which allows to minimize the phase fluctuations between two consecutive observations, i.e., radar measurements. Consequently, an improvement of the displacement measure accuracy is obtained without using complex correction techniques. With low phase noise, the phase variations between two consecutive measurements will be, almost entirely, proportional to the range shift we want to estimate by means of the interferometric algorithm. Precision better than $200 \mu \mathrm{m}$ up to $3 \mathrm{~km}$ distance was obtained [10]. This achievement is strongly welcome for a timely and reliable early detection of structural risks. Moreover, the possibility to replicate the SFCW modulation in several bands (two in our case) allows to highly increase the maximum spectral spacing of the tones, improving the displacement resolution obtained with the interferometric processing [10].

Here, we push forward the study proposed in [10] and extend the one presented in [12], and we investigate the system capabilities in a real multiple target scenario, with the aim to understand how the presence of multiple targets can affect the precision of the single displacement estimates. In this work, we present and discuss both numerical and preliminary experimental results. The obtained results show a good agreement, as it will be discussed in the following of the paper. The outline is as follows: section II introduces the photonics-based radar system, while Section III deals with the theory of differential phase estimation. Sections IV and V present and discuss the numerical simulations and the experimental results, respectively. Finally, Section VI summarizes the main outcomes of the proposed work and recapitulates the results we achieved. Further guidelines are provided as well, which will drive our future research.

\section{The PhOTONICS-BASED RADAR SYSTEM}

The concept of the dual-band photonics-based radar for building integrity analysis and slope stability monitoring applications 


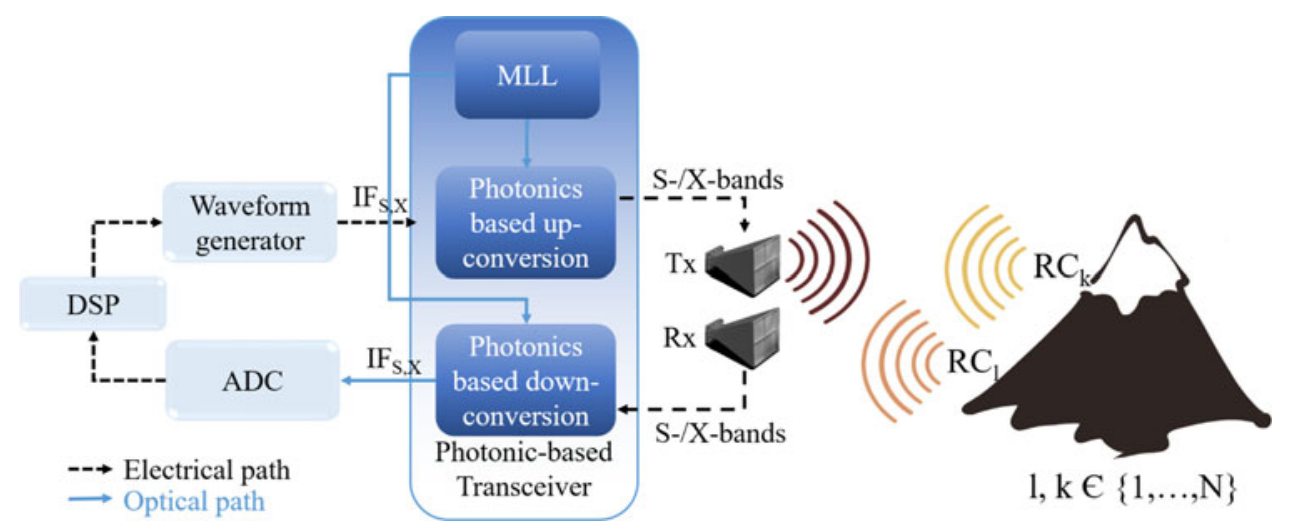

Fig. 1. Concept of the photonics-based dual-band radar system for the monitoring of mountain slope stability and building integrity. In the shaded blue box are included the photonics-based subsystems.

is illustrated in Fig. 1. The radar illuminates the scene transmitting contemporaneously on two separate carrier frequencies, precisely in the S- $(2.475 \mathrm{GHz})$ and $\mathrm{X}$-bands $(9.875 \mathrm{GHz})$. The two transmitted RF signals are generated at intermediate frequencies (IFs) by a digital waveform generator. The IF signals are then fed to the photonics-based transceiver to be upconverted at the RF carrier frequencies. The RF signals, upon amplification by a wideband RF amplifier (WBA), are transmitted towards the targets through a wideband horn antenna. The echoes, collected by a second receiving horn antenna, are first down-converted to IFs by the photonics-based transceiver and then processed by a digital signal processing (DSP) unit.

In our setup, each target lays in a different range cell and can be independently moved through a high precision digitally controlled motorized linear platform. The motorized slide, remotely controlled using a laptop, allows a precise target positioning.

The principle of the photonics-based transceiver is depicted in the shaded blue box in Fig. 1, and detailed in the shaded blue box in Fig. 2 (left). Instead of using RF clocks, a single optical clock is exploited, represented by a mode-locked laser (MLL). The MLL is a highly stable pulsed laser, and its spectrum, as shown in Fig. 2(a), is composed by series of modes spaced by the laser pulse repetition frequency $F_{\mathrm{MLL}}=400 \mathrm{MHz}$. For the frequency up-conversion process, the two signals to be transmitted in S- and X-bands, are generated at $I F_{S}=75 \mathrm{MHz}$ and $I F_{X}=125 \mathrm{MHz}$ respectively, as depicted in Fig. 2(b). Then, they are transferred into the optical domain modulating the MLL optical pulses by means of a Mach-Zehnder modulator (MZM). In fact, using the IF signals as modulating signal, their replicas are generated in the optical domain as lower and upper sidebands of each spectral line of the MLL at IF distance, as shown in Fig. 2(c).

To avoid aliasing, the sum of the $I F_{S}$ and $I F_{X}$ frequencies and their respective spectral occupancy must be chosen less or equal than $F_{\mathrm{MLL}} / 2$. At the output of the MZM, the heterodyning of all the optical spectral components into a photodiode (PD) produces a replica of the two IF signals at every $C F_{S}=k \cdot F_{\mathrm{MLL}} \pm I F_{S}$ and $C F_{X}=l \cdot F_{\mathrm{MLL}} \pm I F_{X}$ frequency, respectively, where $k$ and $l$ are non-negative integers, see Fig. 2(d). At this point, at the output of the PD it is possible to contemporaneously transmit the required up-converted signals at $C F_{S}=2.475 \mathrm{GHz}$ and $C F_{X}=9.875 \mathrm{GHz}$, by using two separate electrical band-pass filters (BPF) locked at the two desired S- and X-band carrier frequencies, as illustrated in Fig. 2(e). Moreover, it is important to observe that the generated carrier frequencies are limited only by the PD bandwidth.

Afterwards, the received RF signal is transferred again to the optical domain, through a second MZM, which modulates the same optical pulse train generated by the MLL. This operation generates replicas of the received RF waveforms around each of the MLL modes. The final spectrum we obtain is, thus, analog to the one represented in Fig. 2(c). Finally, another PD produces a new heterodyning process. In particular, the heterodyning process of each replica with the closest optical carrier produces the down-converted replica of the received RF signals in the starting IF regions. At this point, the signals are filtered by a low-pass filter (LPF), see Fig. 2(f). Then, they are sent to a 400 MSample/s analog-to-digital converter (ADC), which is finally used to digitize both the received and the reference (i.e., the transmitted RF signal) signals for the processing, by means of a digital signal processing (DSP) unit.

\section{DifFERENTIAL PhASE Estimation}

It is possible to precisely determine the target displacement by exploiting a combination of SFCW signal modulation and differential phase measurements [10]. As already mentioned, a radar system which employs SFCW signal modulation transmits a sequence of consecutive coherent sinusoidal signals. The frequency of the $m$ th generic sinusoid is given by:

$$
f_{m}=f_{0}+(m-1) \Delta f
$$

with $m=1, \ldots, N_{\text {step }}$ being the step index, where $N_{\text {step }}$ is the number of sinusoids, $\Delta f$ is the constant frequency separation, namely the frequency step, and $f_{0}$ the carrier frequency, which can assume both the values $C F_{S}$ and $C F_{X}$. Finally, the total signal bandwidth $B W=N_{\text {step }} \Delta f$ defines the achievable range resolution $\Delta R=c /(2 \cdot B W)$.

As known, a variation in the target distance will result in a frequency dependent phase shift of the radar echo. By comparing 


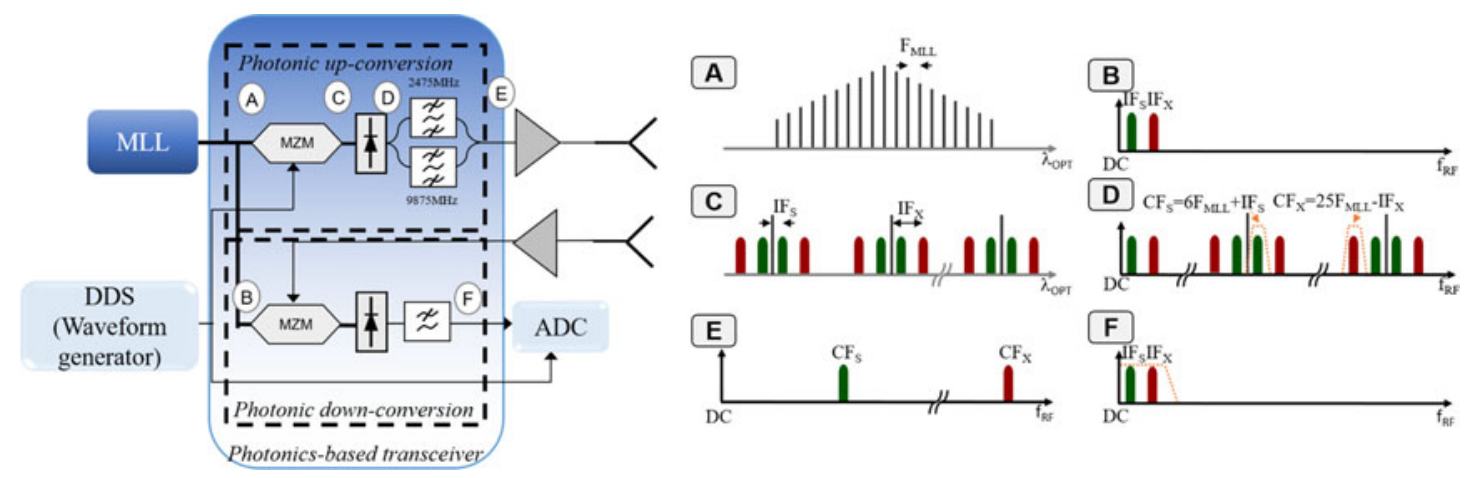

Fig. 2. Scheme of the photonics-based radar system (left), and spectrum in the highlighted point (right). (A) optical spectrum of the mode-locked (MLL) laser used as optical local oscillator; (B) electrical spectrum of the applied radar signals; (C) optical spectrum of the optical modulated signal after the MZM; (D) electrical spectrum at the photodiodes (PD) output; (E) electrical spectrum of the filtered RF radar signals before the wideband antenna; (F) electrical spectrum of the received signals radar at intermediate frequency (IF), after the receiver PD.

the frequency phase of two consecutive echo acquisitions it is possible to determine the target displacement. In fact, each $m$ th frequency component of the SFCW signal accumulates, during the propagation, a phase shift $\Phi_{m}$ proportional to the traveled radial distance. Let us suppose that the radar system transmits at time $t_{0}$, and that the echo signal from a steady target at distance $d$ is collected by the receiver at time $t_{0}+\tau$. Supposing that the propagating medium is just air, the $m$ th sinusoidal signal at frequency $f_{m}$ will accumulate a relative phase contribution $\Phi_{m}$ given by:

$$
\Phi_{m}=2 \pi \cdot f_{m} \cdot \tau=2 \pi \cdot f_{m} \cdot \frac{2 d}{c}
$$

Thus, comparing two consecutive acquisitions of the same $m$ th harmonic, any change $\Delta d_{m}$ in the target distance, can be estimated by the signal phase variation $\Delta \Phi_{m}$ :

$$
\Delta d_{m}=\frac{c}{4 \pi \cdot f_{m}} \cdot \Delta \Phi_{m}
$$

If the phase varies more than $2 \pi$, displacements ambiguities come out, while the maximum unambiguous range $R_{u a}$, can be evaluated as:

$$
R_{u a}=\frac{c}{2 \cdot \Delta f}
$$

By considering (1) and (3), we can observe that a given precision in the phase estimation corresponds to a different accuracy in the range determination, depending on $\Delta f$. The larger is $\Delta f$, the more precise the displacement measure, at the cost of reducing the unambiguous range [13].

If several targets are present in the scene under analysis, and they belong to distinct range cells (i.e., their distance is larger than $\Delta R$ ), we can use standard radar algorithms to process the data. Most implementations use the Inverse Discrete Fourier Transform (IDFT) to transform the data into the spatial domain [14]. By applying proper decomposition algorithms to the received signal, it is possible to determine the separate displacement of each target [15].

Furthermore, as pointed out in [13], the use of large $\Delta f$ allows to detect displacements much smaller than a fraction of millimeter. In our case, higher displacement resolution can be obtained by exploiting the dual-band radar operation. With our setup, it is possible to obtain $N_{\text {step }}$ independent measures with a maximum frequency difference $\Delta f$ up to $7.4 \mathrm{GHz}$ (i.e., the difference between the two selected carriers). Moreover, since the unambiguous displacement is limited to approximately $2 \mathrm{~cm}$, other couples of tones with different $\Delta f$ can be eventually used to resolve ambiguities and to provide a second stage of more precise range evaluation. In this sense, further information can be found in [10], [12].

\section{Simulations}

Numerical simulations were carried out in order to preliminarily demonstrate the effectiveness of the proposed solution. Setup parameters were chosen accordingly to our laboratory equipment availability. In particular, the front-end filters bandwidth is currently limited to $20 \mathrm{MHz}$, thus forcing us to limit the SFCW signal bandwidth to $B W \leq 20 \mathrm{MHz}$.

By imposing the maximum unambiguous range $R_{u a}$ equal to $500 \mathrm{~m}$, it is possible to retrieve from (4) the frequency step $\Delta f$, which is the analog of the pulse repetition frequency (PRF) in pulse radar nomenclature [2]. In this way, given the total system bandwidth limited to $B W=20 \mathrm{MHz}$, the number of frequency steps can be retrieved as $N_{\text {step }}=B W / \Delta f=66$. Thus, the maximum unambiguous range $R_{u a}$ is divided into $N_{\text {step }}$ range cells with extension $\Delta R$. In order to obtain a range resolution $\Delta R=7.5 \mathrm{~m}$, the duration $T_{\text {step }}$ of each step must be equal to $50 \mathrm{~ns}$, in other words $T_{\text {step }}=1 / B W$. These parameters were set common to both the carrier frequencies, resulting in two identical coherent sequences of SFCW signals being transmitted in the S- and X-bands.

All the radar setup parameters are summarized in Table I, while five targets were simulated simultaneously at 25,145 , 220,300 and $400 \mathrm{~m}$ distance from the radar. As already said, in our case for simplicity, but without loss of generality each target is in a different range cell.

Each target is characterized by its own arbitrary displacement pattern, as depicted in Fig. 3. However, in a real scenario the displacement independence assumption among confining range cells will be not necessarily true. Let us think, for instance, to the 
TABLE I

Setup Parameters of the S- AND X-BAnd SFCW Signals TransmitTed BY THE PHOTONICS-BASED RADAR SYSTEM

\begin{tabular}{ccc}
\hline Parameter & S-band & X-band \\
\hline Intermediate Frequency & $75 \mathrm{MHz}$ & $125 \mathrm{MHz}$ \\
$I F_{S}, I F_{X}$ & $6 F_{M L L}+I F_{S}$ & $25 F_{M L L}-I F_{X}$ \\
Carrier Frequency & $2.475 \mathrm{GHz}$ & $9.875 \mathrm{GHz}$ \\
$C F_{S}, C F_{X}$ & $400 \mathrm{MHz}$ \\
Sampling Frequency $F_{M L L}$ & $300 \mathrm{kHz}$ \\
Frequency step $\Delta f$ & 66 \\
No. of steps $N_{s t e p}$ & $\approx 20 \mathrm{MHz}$ \\
Bandwidth $B W$ & $50 \mathrm{~ns}$ \\
Time step & \multicolumn{2}{c}{1000} \\
duration $T_{\text {step }}$ & \multicolumn{2}{c}{15} \\
No. of SFCW periods & \\
per data record & \\
No. of data records &
\end{tabular}

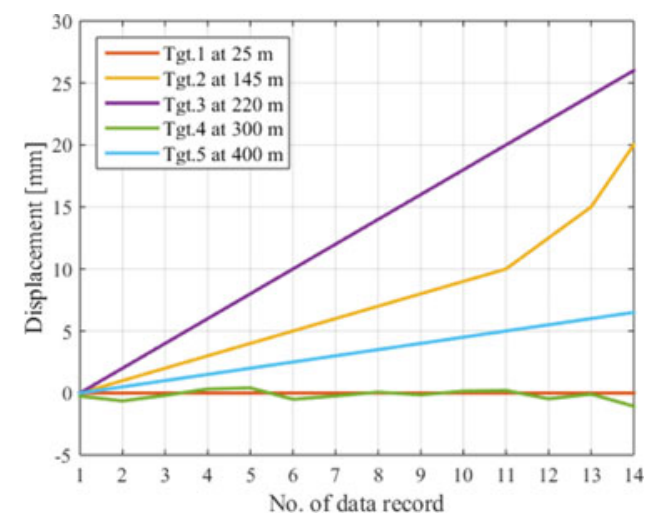

Fig. 3. Simulated displacement curves versus the data record index. Five targets are considered at 25 (red), 145 (yellow), 220 (purple), 300 (green) and $400 \mathrm{~m}$ (cyan) distance from the radar, respectively. Displacements are assumed to be independent.

structural movements along the side of a mountain. Moreover, different values of radar cross section (RCS) values were considered to simulate real and different targets (e.g., walls, slopes, bridges, etc.).

The processing chain undergone by the received IF signals $s(t)$, respectively at $I F_{S}$ and $I F_{X}$ frequencies [see Fig. 2(f)], where $t$ denotes the time dependence, is illustrated Fig. 4. However, in the proposed scheme, the dependence on the carrier frequency is omitted for brevity.

First, the cross-correlation is carried out between the received signal $s(t)$ and the reference signal $r(t)$ to evaluate the presence of possible targets and their distance. In the plot on the left of Fig. 4 the obtained cross-correlations are shown for the five simulated targets, exactly centered at their respective range distances. At the same time, the mixed signal is evaluated in the time domain as $m(t)=r(t) \cdot s^{*}(t)$, where * is the complex conjugate operator. From the analysis of the cross-correlation peaks, once the presence of a target and its distance from the radar are determined, the phase of $M(f)$, i.e., the Discrete Fourier Transform (DFT) of $m(t)$, is evaluated for those spectral components corresponding to the estimated ranges. $M(f)$ is depicted in the right plot of Fig. 4. By comparing two successive phase measurements, the target displacement can be determined by applying (3).
The simulated results are depicted in Fig. 5, which shows the error curves obtained from the true and the estimated displacements for the five simulated targets in the S-band. In the simulations, fifteen sets of measurements are generated, each consisting of 1000 pulse repetitions of the SFCW signal. The first record is taken as a reference for the successive phase calculations. The indexes of the data records are plotted along the abscissas in Fig. 5. As we can observe, the estimation error is usually smaller, in modulus, than $0.2 \mathrm{~mm}$ for all the five simulated targets up to $400 \mathrm{~m}$. However, it is worthy to observe that the estimated displacements are also slightly influenced by the relative positions of all the targets in the scene. This is clear for targets 4 and 5, at 300 and $400 \mathrm{~m}$ and depicted by the green and blue curves respectively, for which some degree of correlation can be observed between the two.

A further analysis is conducted considering different values of signal-to-noise ratio (SNR) affecting the received signal. Without loss of generality, with the term noise we can indicate both the system noise and the clutter collected from the scene under analysis. In this study, a complex white Gaussian noise has been considered for simplicity.

The results of the numerical simulations are shown in Fig. 6. The error curves between the true and the estimated displacement are estimated only for the second target (i.e., the one at $145 \mathrm{~m}$ ). As for the previous analysis, fifteen sets of measurements are generated, each composed of 1000 pulse repetitions of the SFCW signal. The real target displacement behavior is depicted by the ochre curve in Fig. 3. The final evaluated displacement is estimated through differential phase measurements between the $\mathrm{S}$ - and $\mathrm{X}$-bands, therefore, considering a larger $\Delta f$ of $7.4 \mathrm{GHz}$ between the two S- and X-band carriers [10]. It can be observed that the absolute mean error is well below the millimeter threshold, for positive SNR values larger than about $10 \mathrm{~dB}$. Below this value, the error rapidly increases because the useful signal becomes even more submerged in noise. This event is comparable to the situation in which the target RCS is smaller than the noise RCS. A possible solution to avoid this problem could be to increase the radar range resolution capability (i.e., decrease $\Delta R$ ) and have a finer angular resolution (e.g., using a more directive antenna or synthesizing a more directive beamwidth through SAR processing). At the current moment, these possibilities are actually under further investigation.

\section{EXPERIMENTAL RESULTS}

A preliminary experiment was conducted to prove the effectiveness of the photonics-based transceiver for detecting and measuring displacements when multiple targets are present. In the experiments the same radar system parameters reported in Table I were used. The only exception was the length of each acquisition record, which was set equal to 100 repetition periods of the SFCW waveform. Two metal surfaces of about $0.25 \mathrm{~m}^{2}$ were considered as targets. The first target was fixed at $25 \mathrm{~m}$ away from the radar and it was used as a phase reference. The second target, placed at $145 \mathrm{~m}$, was moved with $1 \mathrm{~mm}$ steps from 0 to $100 \mathrm{~mm}$. For completeness, the spectrum of the received SFCW signal at the output of the ADC, and integrated over 1000 


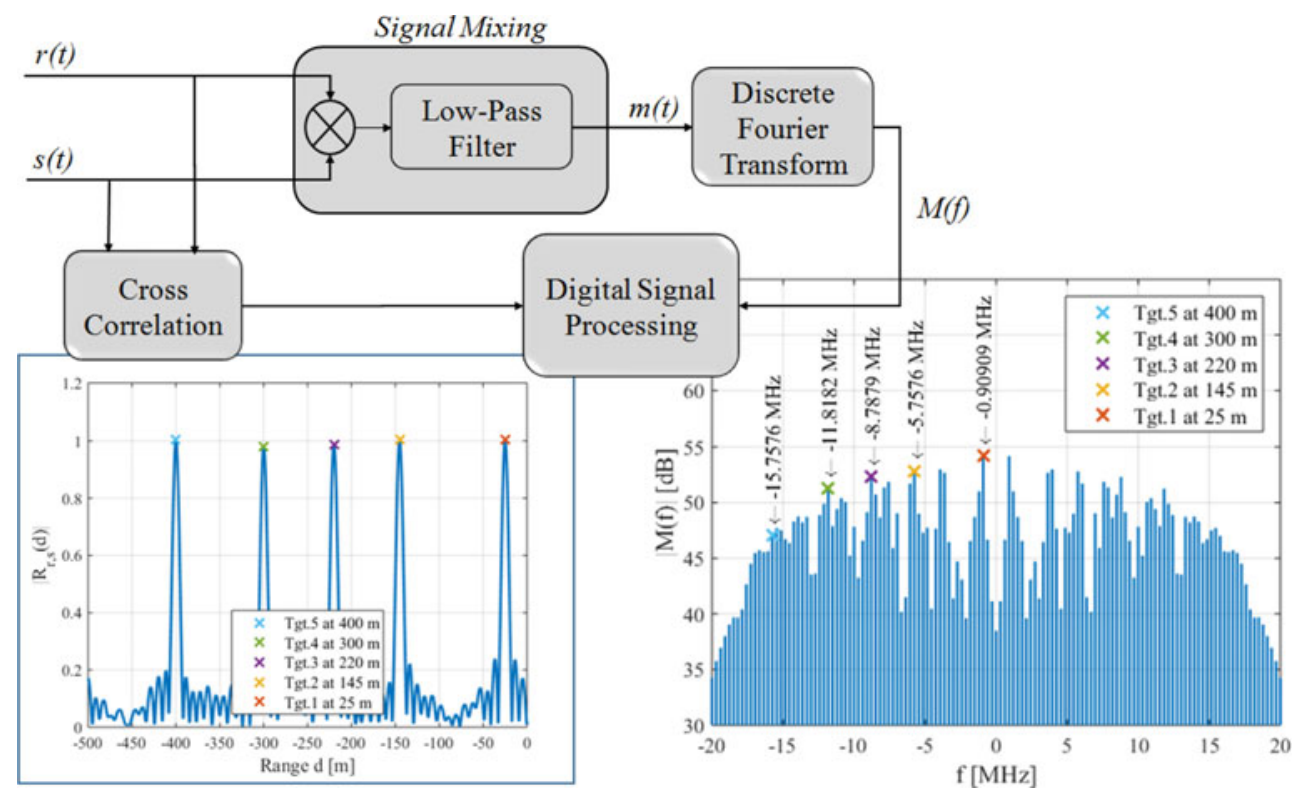

Fig. 4. Processing chain undergone by the received IF signals $s(t)$, respectively at $I F_{S}$ and $I F_{X}$ frequencies. The cross-correlation between $s(t)$ and the reference signal $r(t)$ is reported on the left, while $M(f)$ is reported on the right.

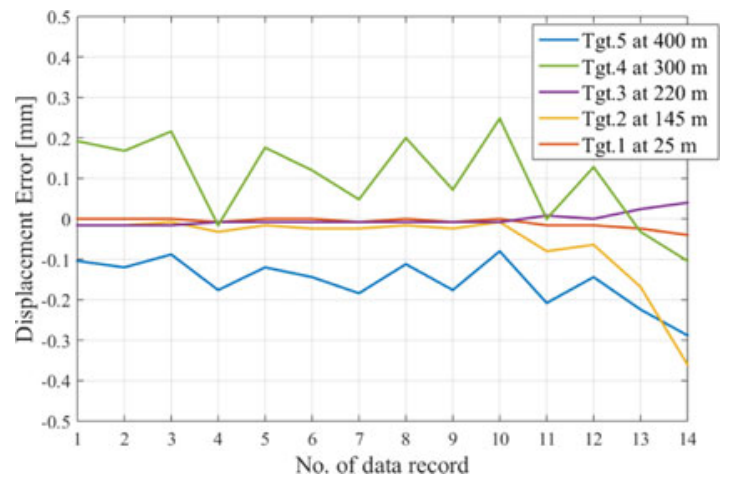

Fig. 5. Error curves after the displacement estimation for the five simulated targets. Only the S-band radar data are shown.

SFCW waveform repetition periods, is depicted in Fig. 7. The S-band signal, centered at $I F_{S}= \pm 75 \mathrm{MHz}$, and the X-band signal, centered at $I F_{X}= \pm 125 \mathrm{MHz}$, can be observed; each of them is composed by 66 frequency steps.

The actual and the estimated displacements of the target are shown in Fig. 8(a). One hundred acquisitions were processed and, in each of them, the target at $145 \mathrm{~m}$ from the radar was moved with steps of $1 \mathrm{~mm}$. As depicted in Fig. 8(a), a good agreement can be found between the actual and the measured displacements. Fig. 8(b) depicts the accuracy of the system, which is defined as the difference between the actual and the estimated displacement. For this set of measurements the maximum error found was $0.25 \mathrm{~mm}$, which indicates a good agreement with the numerical simulations. Therefore the presence of multiple scatterers did not worsen the performance of the photonics-based system w.r.t. the single target case, reported in [10]. It can be noted that the experimental accuracy was

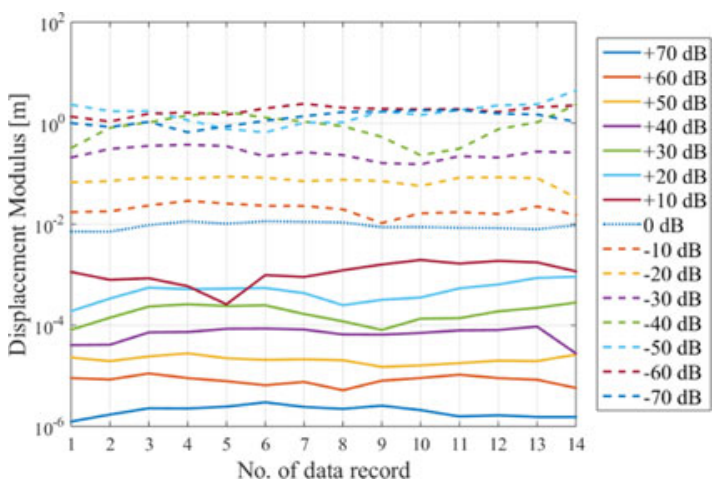

Fig. 6. Absolute values in log-scale of the displacement estimation error at the varying of the SNR, for the simulated target at $145 \mathrm{~m}$ distance. Displacement estimates are obtained by exploiting both S- and X-bands.

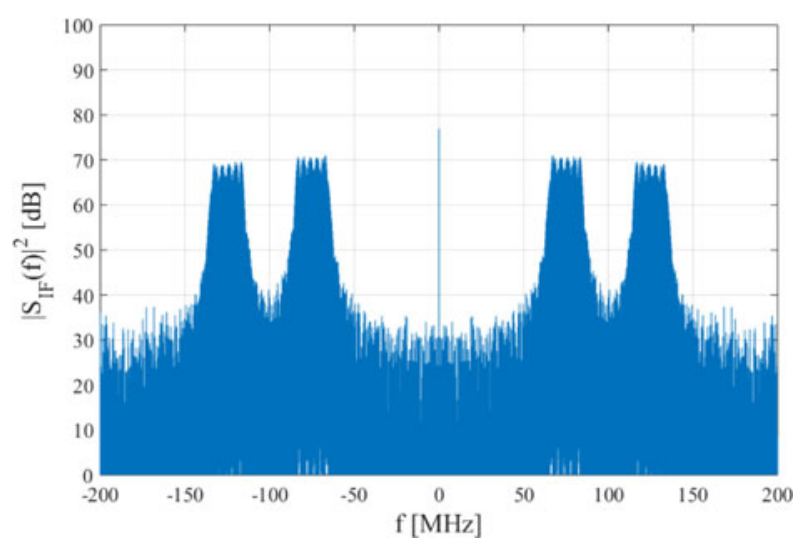

Fig. 7. Electrical spectrum of the received SFCW signal after IF downconversion. The two (specular) signal components at $I F_{S}=75 \mathrm{MHz}$ and $I F_{X}=125 \mathrm{MHz}$ can be observed. 


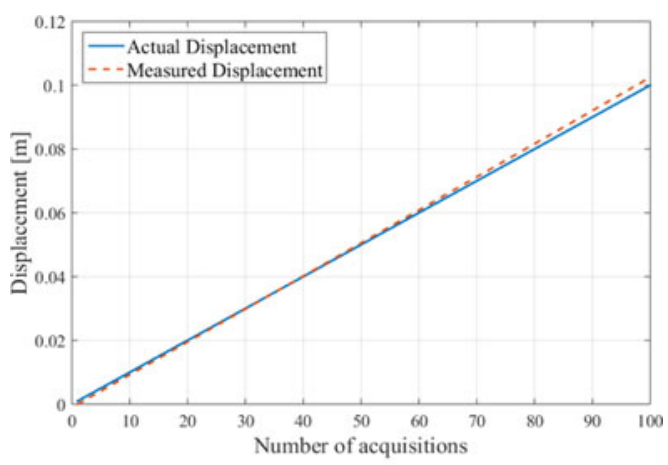

(a)

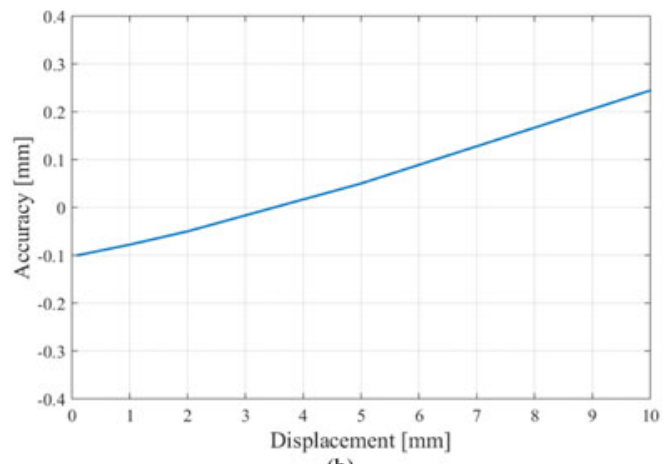

(b)

Fig. 8. Preliminary results obtained with the real signal at the output of the ADC: (a) True (blue line) and measured (red dashed line) displacements; (b) Accuracy as a function of the displacement.

slightly worse than in the simulations, since, in the last case, the noise sources of the phonics-based up- and down-conversion stages were not taken into account.

\section{CONCLUSIONS}

In this work, a dual-band photonics-based radar system used for precise displacement measurements in a multi-target scenario was presented. By pushing forward our previous work, in which we analyzed the same system for displacement measures of a single target, here we analyze the impact of the presence of several scatterers on the single displacement measurements. $\mathrm{Nu}-$ merical simulations were realized by simulating five different targets at different ranges with different RCS values. A precision lower than $0.2 \mathrm{~mm}$ for distances up to $400 \mathrm{~m}$ was obtained, as in the case of the single target. It was observed that this precision slightly depends on the relative positions of the targets. In addition, an accurate analysis, for different operative conditions of SNR was carried out.

The experimental results showed a good agreement with the simulated data. We considered two targets placed at two different positions, respectively at 25 and $145 \mathrm{~m}$ from the radar. We found a maximum error of $0.25 \mathrm{~mm}$, proving that the system can be exploited with success for interferometric measurements also in the presence of multiple targets. A potential real-case scenario is the monitoring of areas subject to possible landslides, or the monitoring of building integrity, where multiple scatterers, also referred to as targets, are present in the scene under analysis.
The use of a single photonics-based transceiver for handling the signals in both the RF bands ( $S$ and $X$ ) with high degree of coherence allows to obtain high displacement accuracy, with sub-mm resolution, with no need of further correction algorithms. The use of multiple bands adds, among the numerous advantages, robustness to the environmental conditions and it allows to exploit harmonics with very large frequency separation.

\section{REFERENCES}

[1] D. Tarchi, "Monitoring landslide displacements by using ground-based synthetic aperture radar interferometry: Application to the Ruinon landslide in the Italian Alps," J. Geophys. Res., vol. 108, no. B8, 2003. [Online]. Available: http://doi.wiley.com/10.1029/2002JB002204

[2] M. I. Skolnik, Radar Handbook, 3rd ed. New York, NY, USA: McGrawHill Education, Feb. 2008.

[3] X. Shi, M. Liao, M. Li, L. Zhang, and C. Cunningham, "Wide-Area landslide deformation mapping with multi-path ALOS PALSAR data stacks: A case study of three gorges area, China," Remote Sensing, vol. 8, no. 2, Feb. 2016, Art. no. 136. [Online]. Available: http://www.mdpi.com/20724292/8/2/136

[4] A. Meta, P. Hoogeboom, and L. P. Ligthart, "Signal processing for FMCW SAR," IEEE Trans. Geosci. Remote Sens., vol. 45, no. 11, pp. 3519-3532, Nov. 2007. [Online]. Available: http://ieeexplore. ieee.org/document/4373378/

[5] M. Marchisio, L. Piroddi, G. Ranieri, S. V. Calcina, and P. Farina, "Comparison of natural and artificial forcing to study the dynamic behaviour of bell towers in low wind context by means of ground-based radar interferometry: The case of the Leaning Tower in Pisa," J. Geophys. Eng., vol. 11, no. 5, 2014, Art. no. 055004. [Online]. Available: http://stacks.iop.org/1742-2140/11/i=5/a=055004

[6] I. Nicolaescu, P. van Genderen, K. Van Dongen, J. van Heijenoort, and P. Hakkaart, "Stepped frequency continuous wave radar-data preprocessing.," Int. Res. Centre Telecommun. Transmiss. Radar, 2003, pp. 177-182. [Online]. Available: http://ieeexplore.ieee.org/document/1207315/

[7] A. B. Suksmono, E. Bharata, A. A. Lestari, A. G. Yarovoy, and L. P. Ligthart, "Compressive stepped-frequency continuous-wave groundpenetrating radar," IEEE Geoscience Remote Sens. Lett., vol. 7, no. 4, pp. 665-669, Oct. 2010. [Online]. Available: http://ieeexplore. ieee.org/document/5454278/

[8] P. Ghelfi et al., "A fully photonics-based coherent radar system," $\mathrm{Na}$ ture, vol. 507, no. 7492, pp. 341-345, Mar. 2014. [Online]. Available: http://www.nature.com/doifinder/10.1038/nature13078

[9] F. Scotti, F. Laghezza, P. Ghelfi, and A. Bogoni, "Multi-Band softwaredefined coherent radar based on a single photonic transceiver," IEEE Trans. Microwave Theory Techn., vol. 63, no. 2, pp. 546-552, Feb. 2015. [Online]. Available: http://ieeexplore.ieee.org/document/7006701/

[10] S. Pinna, S. Melo, E. Lazzeri, A. Bogoni, F. Scotti, and F. Laghezza, "Submm displacement measure via multi-band phase estimation in a photonicsbased radar system," in Proc. Eur. Radar Conf., 2016, pp. 310-313. [Online]. Available: http://ieeexplore.ieee.org/abstract/document/7811709/

[11] J. D. Taylor, Ed., Ultra-Wideband Radar Technology. Boca Raton, FL, USA: CRC Press, 2001.

[12] S. Melo et al., "High precision displacement measurements in presence of multiple scatterers using a photonics-based dual-band radar," in Proc. IET Radar Conf., Belfast, U.K., Oct. 2017, p. 6.

[13] K. Meiners-Hagen, R. Schdel, F. Pollinger, and A. Abou-Zeid, "Multiwavelength interferometry for length measurements using diode lasers," Meas. Sci. Rev., vol. 9, no. 1, pp. 16-26, Jan. 2009. [Online]. Available: http://www.degruyter.com/view/j/msr.2009.9.issue-1/v10048009-0001-y/v10048-009-0001-y.xml

[14] A. Langman and M. R. Inggs, "Pulse versus stepped frequency continuous wave modulation for ground penetrating radar," in Proc. 2001 Int., Geoscience Remote Sens. Symp., 2001, pp. 1533-1535.

[15] H. Zebker and J. Villasenor, "Decorrelation in interferometric radar echoes," IEEE Trans. Geosci. Remote Sens., vol. 30, no. 5, pp. 950-959, Sep. 1992. [Online]. Available: http://ieeexplore.ieee.org/ document/175330/ 